\title{
UML-based combat effectiveness simulation system modeling within MDE
}

\author{
ZHU Zhi ${ }^{1,2, *}$, LEI Yonglin ${ }^{1,2}$, SARJOUGHIAN Hessam² ${ }^{2}$ LI Xiaobo ${ }^{1}$, and ZHU Yifan ${ }^{1}$ \\ 1. Department of Military Modeling and Simulation, School of Systems Engineering, National University of Defense Technology, \\ Changsha 410073, China; \\ 2. Arizona Center for Integrated Modeling and Simulation, School of Computing, Informatics, and Decision Systems Engineering, \\ Arizona State University, Tempe 85281, USA
}

\begin{abstract}
To reduce complexity, the combat effectiveness simulation system (CESS) is often decomposed into static structure, physical behavior, and cognitive behavior, and model abstraction is layered onto domain invariant knowledge (DIK) and application variant knowledge (AVK) levels. This study concentrates on the specification of CESS's physical behaviors at the DIK level of abstraction, and proposes a model driven framework for efficiently developing simulation models within model-driven engineering (MDE). Technically, this framework integrates the four-layer metamodeling architecture and a set of model transformation techniques with the objective of reducing model heterogeneity and enhancing model continuity. As a proof of concept, a torpedo example is illustrated to explain how physical models are developed following the proposed framework. Finally, a combat scenario is constructed to demonstrate the availability, and a further verification is shown by a reasonable agreement between simulation results and field observations.
\end{abstract}

Keywords: domain specific modeling, model-driven development, system engineering, effectiveness simulation.

DOI: $10.21629 /$ JSEE.2018.06.07

\section{Introduction}

Many of today's combat systems often combine multiple subsystems, e.g., a platform for engage-level simulations typically combines a command and control (C2) center, sensors, weapons, and a variety of countermeasures. Each subsystem may span multiple disciplines, e.g., the sensor subsystem may include radar, sonar, laser, infrared detectors, etc. Specifying such complex systems is quite challenging, requiring not only good hierarchical architecture, but also a combination of modeling paradigms and formalisms.

To reduce system specification complexity, combat sys-

Manuscript received August 11, 2017.

*Corresponding author.

This work was supported by the National Natural Science Foundation of China (61273198). tems are decomposed into three closely related but mutually isolated aspects: static structure, physical behavior, and cognitive behavior; domain-level invariant knowledge (DIK) and application-level variant knowledge (AVK) are identified to represent different levels of details [1]. Other methodologies include combining multiple views of a given system, e.g., functional and non-functional properties, and appropriate use of related models at distinct stages of the development lifecycle, e.g., requirement analysis, conceptual modeling, simulation model development, experimentation, and result analysis [2].

Such considerations raise various problems, such as model heterogeneity, and the lack of model continuity when various paradigms and formalisms are used to represent different models at different modeling stages. Furthermore, in the model driven engineering (MDE) context, the number of modeling languages tends to increase with the benefits of meta-modeling and model transformation techniques. These issues challenge the methodologies and tools that simulation developers use to specify, design, simulate, and analyze systems. It is not sufficient to adopt a generic simulation platform that realizes one or few modeling paradigms, neither is it sufficient to create a dedicated simulation system for a specific type of combat effectiveness simulation system (CESS). Rather, the ideal is to explore a unified method to reduce model heterogeneity and enhance model continuity.

This study proposes a model-driven framework for CESS modeling within MDE. This framework provides a formal approach to guide development of CESS models by integrating metamodeling and model transformation techniques. It addresses model heterogeneity caused by combining modeling languages as well as model continuity caused by semantic gaps between different modeling stages. In contrast to heavily weighted metamodeling methods, such as eclipse modeling framework (EMF) 
[3], unified modeling language (UML) profile as a lightly weight method is adopted because a wide range of modeling resources (e.g., modeling tools and tutorials) are available, and it provides a common foundational language infrastructure. In addition, the framework concentrates on CESS's physical behaviors specification at the DIK level of abstraction. However, it can also be incorporated into existing methodologies for developing the static structure and cognitive behavior.

The remainder of this paper is structured as follows. Section 2 introduces the background and related work. Section 3 presents the model-driven framework. In Section 4 , a physical domain specific profile is designed based on the proposed framework and a case example is also designed using the profile. After that, Section 5 demonstrates the example by constructing a simulation application, which is followed by conclusions in Section 6 .

\section{Background and related work}

Due to inconsistent terminology for CESS, it is necessary to define basic meanings of important frequently used terms to provide a common understanding. Many of these terms are used alternatively in specific contexts, but providing their definitions and/or subtle distinctions is helpful to understand the methodologies, techniques, and tools.

\subsection{What is CESS?}

CESS is featured with multidiscipline and integrative knowledge, leading to its design quite challenging today. For this reason, the design generally requires: first, a separation of concerning domains that have different levels of flexibility; second, a domain knowledge abstraction at different levels; third, an abstract, formal, and platformindependent language tailored in a more understandable and modifiable way; last, a set of constraints that are enforced at a higher level of abstraction.

\subsubsection{Static structure, physical and cognitive behaviors in CESS}

Static structure contains a number of inherent entities and relations. It is the most stable part and is determined by the nature of a given system. UML class diagrams, entityrelationship diagrams (ERD) and system entity structure (SES) are commonly used to represent this part. Ontologies are also used to capture system structural concepts [4].

Physical behavior refers to the dynamic behavior of a specific entity regarding the physical and information aspects. It is relatively stable but in a somewhat variant form across different applications. General-purpose modeling techniques such as dataflow, process networks, rendezvous, synchronous-reactive models, finite state machines, and discrete event models, are employed. Others include domain specific languages (DSL), such as business process modeling notation (BPMN) [5], a conceptual modeling approach KAMA [6], and simulation model definition language (SMDL) [7].

Cognitive behavior belongs to the cognitive and social domain where human will or outside environment plays key roles. Due to the difference between decision maker abilities, cultural traditions, and educational background, it is most flexible thus usually occurs as scripts. Hence, a similar simulation model can be reused to schedule multiple cognitive scripts through predefined interfaces, i.e., script based tactics managers [8]. To capture the overall process that human beings or organizations use to think, grow, and thrive in rapidly changing environments, the observe orient decide act (OODA) loop methodology [9] is generally well suited for explicitly specifying ambiguity and uncertainty.

\subsubsection{DIK and AVK}

DIK and AVK are identified from the perspective of model abstraction. The former is built through the 'abstraction' mechanism by hiding details while the latter is built through the 'refinement' mechanism by adding details.

DIK represents the commonality and generality at a higher level of abstraction, e.g., the fundamental concepts and properties of a given system. Although a general modeling and simulation technique, such as simulation protocol like high level architecture (HLA) [10], model specification like simulation modeling platform (SMP2) [11], and model formalism like UML [12], is crucial to specify DIK, experiences show that constructing a set of composable modeling frameworks [13] is the key to DIK engineering.

AVK extends or customizes DIK at a lower level of abstraction. Therefore, the definition principles are similar to DIK, except for the system application. Its representation is usually achieved through general-purpose programming languages, such as $\mathrm{C}++$, Python, etc., in the form of dynamic linkage libraries (DLLs).

\subsection{CESS modeling methodologies}

\subsubsection{Typical stages of model development}

There have been many attempts to increase the probability of success in modeling and simulation $(M \& S)$ studies by proposing common modeling and simulation processes, which are composed of several stages. From the detailed and comprehensive M\&S lifecycle [14], model development and execution lifecycle and related infrastructure support for CESS are provided to guide developers for various objects, activities, and infrastructure [15]. Although the specific context of a given application may ap- 
pear different, most well-known M\&S processes emphasize conceptual modeling, specification, and implementation.

(i) Conceptual modeling elicits general knowledge about a problem/research domain and describes a conceptual model [16]. In this stage, the stakeholder and conceptual modeler are both important to produce a conceptual model to be consumed in subsequent stages. This model is non-executable, i.e., there is no algorithm to compute model behavior according to the semantics of the modeling language employed [17].

(ii) Model specification is a detailed description of required work or materials to be used to describe the formal model according to a special formalism, such as discrete event system specification (DEVS), Petri net, or partial differential equations. This stage transforms the conceptual model into a formal model whose behavior can be computed or executed, but without considering the specific platform.

(iii) Model implementation produces a computer model that can be simulated on a specific platform. However, this model must be transformed to a final compiled DLL or executable source code. The computer and formal model may be viewed as simulation models in a more encompassing meaning when discussing M\&S lifecycles.

\subsubsection{Related modeling techniques}

Many terms are used to express models at different levels of precision for different purposes, including paradigm, formalism, model of computation (MoC), and language. For a given domain, some of them can be used interchangeably, thus it is important to know their subtle distinctions, as shown in Fig. 1.

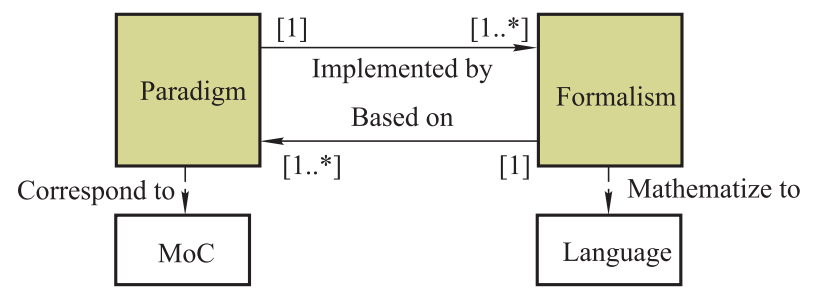

Fig. 1 Relationships between modeling techniques

A modeling language is used to formally represent a model in a specific syntax and semantics [18]. Syntax has abstract and concrete aspects. Abstract syntax defines the set of syntactically correct models that describe modeling language concepts, relationships, and well formedness rules. Concrete syntax provides the notation to construct and present a model, which can be textual and/or visual: textual syntax uses constructed text to represent models, whereas visual syntax represents the language diagrammatically.
Semantics describes the language meaning and also has two types. Static semantics defines the well formedness of the constructs in a language in the form of invariant conditions that must hold for any model created using the language. Dynamic semantics is the interpretation of a given set of constructs in the specific context.

A modeling language can be considered a modeling formalism with a formal syntax and semantics, where 'formal' means 'mathematical', i.e., formalism mathematizes the language. However, a mathematic definition may be useful but is not necessary [19]. Modeling formalism is mostly used to represent simulation models, whereas a modeling language is mostly used to express conceptual models, e.g., Petri net is a modeling formalism whereas UML is a modeling language.

A modeling paradigm refers to a set of requirements that governs how any system in the particular domain is to be modeled [20]. More specifically, we consider that a modeling paradigm is a distinct set of concepts, thoughts, or patterns, including modeling theories, methods, postulates, and standards that constitute legitimate contributions to a system/domain. The discrete event model, for example, is a modeling paradigm, and can be implemented by several formalisms, e.g., DEVS-C++ [21]. A modeling formalism/language can be based on multiple paradigms. This usually happens in a generic formalism where two or more paradigms are jointly realized, e.g., a new language can be constructed based on finite state machines and its timed version to express concepts such as timeouts and delays.

$\mathrm{MoC}$ is a more detailed and accurate version of the modeling paradigm. In many cases, a modeling paradigm corresponds to a MoC. This term [22] provides a collection of rules that govern concurrent execution of components and communication between components, defining what constitutes a component, execution and concurrent mechanisms, and communication mechanisms. Ptolemy II [23] is featured with two important concepts: domain and director. In fact, a director is a block that specifies the $\mathrm{MoC}$, which is a realization of a domain, and a domain is an implementation of a director, i.e., MoC. Note this paper uses the term modeling technique to encompass modeling language, formalism, paradigm, or MoC.

\subsubsection{Model heterogeneity and model continuity}

Model heterogeneity is the level of dissimilar or diverse elements, relationships, or well-formedness rules between models that represent the same system at a given point in the development process. Heterogeneity is generally unavoidable and may even be essential, because using different modeling techniques to better describe global system properties, particularly for complex systems, is required 
to suit different modeling goals. Hence, the key is methodologies or techniques to integrate heterogeneous models, such as multi-paradigm modeling (MPM) [24].

Model continuity refers to the generation of an appropriate morphism relation between the different models of a development process through predefined transformation rules. Unlike model heterogeneity, lack of model continuity can be avoided if the predefined formal transformation rules are sufficient to support effective consummation of the initial and intermediate models in later development stages and to preserve the semantics $[25,26]$. Hence, the key to obtain model continuity is to design formal rules of model transformations.

Model heterogeneity and continuity generally have different causes. Heterogeneity arises in a collection of models expressed in different modeling techniques (languages or paradigms), whereas continuity problems occur due to large semantic gaps between different models (initial, intermediate, and final code) during the development process.

\subsection{CESS modeling techniques}

Current approaches for engineering CESS have different classifications. From the perspective of M\&S lifecycle, for example, Li et al. [15] introduced unified formalism (e.g., DEVS [27], Modelica [28]), unified model specification (e.g., base object model (BOM) [29] and SMP2 [30]), unified composable framework (e.g., EADSim [31] and weapon effectiveness simulation system (WESS) [32]), and unified simulation protocol (e.g., HLA [10]) based methods, then proposed a model framework based domain specific composable modeling method to realize composable and multi-domain modeling techniques.

Generally, to satisfy non-functional requirements (i.e., model composability, domain specific modeling, and model evolvability), modeling methods are classified from domain neutral, application specific, and domain oriented perspectives. Domain neutral or application specific perspectives provide powerful infrastructure with many generic $M \& S$ techniques embedded and a model library containing many domain components. Hence, simulation applications are easily built in the form of fixed infrastructure plus components. In this approach, domain knowledge is not adequately expressed. In domain oriented simulation systems, different simulation applications can be composed from built-in components and configured with application specific parameter values, such as JointMEASURE [33].

These methods are widely used among researchers and engineers in the M\&S field. Some concentrate on addressing general technical problems, whereas others are promi- nent and typical for CESS engineering. In either case, a wide range of model heterogeneity and model continuity exists. We compare the properties and capabilities of these methods in terms of model heterogeneity and continuity support with four levels (well, medium well, medium, low), as shown in Table 1. For brevity, we number the formalism, specification, composable framework, simulation protocol, and domain oriented systems as method 1 , method 2 , method 3 , method 4 , and method 5 , respectively.

Table 1 Capabilities of methods regarding model heterogeneity and model continuity

\begin{tabular}{cccc}
\hline Number & Example & Heterogeneity & Continuity \\
\hline 1 & DEVS, Modelica & Well & Well \\
2 & BOM, SMP2 & Well & Medium well \\
3 & EADSim, WESS & Medium well & Medium \\
4 & HLA & Low & Low \\
5 & JointMEASURE & Medium & Medium \\
\hline
\end{tabular}

The heterogeneity difficulty level depends on jointly used formalisms and paradigms. Using different formalisms with different paradigms, for example, produces the most difficult level of heterogeneity (method 4), whereas similar formalisms with similar paradigms produce the lowest level of difficulty, also called homogeneity (methods 1 and 2). Similar formalism with different paradigms or similar paradigms with different formalisms produces medium level of difficulty (method 5), but in a somewhat milder form if a unified composable framework is used $(\operatorname{method} 3)$.

In contrast to heterogeneity, continuity mainly depends on the quality of the model transformation rules. The formalism based method 1 exhibits the best model continuity because the transformation model has formal syntax and semantics that are precisely and unambiguously defined. The specification based method 2 exhibits the second level of model continuity since the transformation model design complies with a similar model specification. Since there is little relationship between a composable framework and model continuity, method 3 and method 5 share the same medium continuity level. The simulation protocol based method 4 exhibits the lowest level of model continuity, because the main purpose of a simulation protocol is to support simulation level interaction and interoperability of different simulation components, promoting simulation resource reuse, rather than the model level purpose.

Focusing on CESS modeling, another classification includes general purpose and domain specific modeling techniques. We introduce three representative general purpose modeling techniques as well as some related domain specific modeling techniques.

A typical general purpose modeling technique is dataflow: either static synchronous dataflow (SDF) or dynamic dataflow (DDF) [34]. This technique is appropri- 
ate for applications that involve processing data streams, which can flow through actors that transform them in some way. One well-known SDF mode is the pipe and filter. Raw data is carried by pipes and flows through sequences of filters to provide the required data. In CESS, we consider screening incoming threats as an example, and design a composite filter containing concrete filters, such as sizeFilter and RCSFilter, to obtain a desired target that falls within the specified range of size and radar cross section (RCS). Another typical mode is the chain of responsibility. A request is processed through a sequence of actors who are in charge of and will not be approved until the final actor handles. One prominent example is that a request for attack action is handled by layers of $\mathrm{C} 2$ centers. This action cannot start until the very top commander approves it.

Finite state machine (FSM) is another commonly accepted and widely used paradigm to model physical behavior. An FSM contains a finite set of states and rules that govern transitions between them. The set of states has one initial state and any number of final states. Each transition has a guard expression, any number of output actions, and any number of set actions. FSM execution starts with an initial state and executes a sequence of steps upon each firing. A number of physical models in CESS have been effectively expressed as FSMs, e.g., wire guided torpedo behavior incorporates a set of states, such as wire guided, homing guided, final attack, and helical search. Once the radiated acoustic signal is interrupted during the wire guided state, the torpedo opens its sonar and enters the homing guided state.

Ptera [35], an event oriented paradigm, is appropriate to specify timed behaviors where input events may trigger chain reactions. In contrast to general modeling graphs (e.g., activity diagrams) where transitions are utilized as events to trigger activity changes, Ptera components are events, and transitions between events are expressed as triggering relations. An example Ptera model is multiple target assignments of a single platform equipped with multiple weapons. A platform has a target list shared by the armed weapons. When a target is within the platform range, it is placed at the end of the target list to await assignment using the first input first output (FIFO) strategy. When a target is assigned, the target list size is decreased by 1 as well as the weapon list. When the assignment is completed, the weapon list is decreased by 1 , but the target list size may remain unchanged if the task fails.

There are three primary methods to define domain specific modeling techniques (languages): refine an existing general modeling language, specializing some of its general constructs to represent domain specific constructs; extend an existing modeling language, supplementing with fresh domain specific constructs; and define a new modeling language from scratch. There is no definite best selection, because each method has advantages and drawbacks.

The last technique seems most suitable for defining a DSL, since the essence of domain specific modeling (DSM) is direct modeling with familiar domain concepts, relationships, and constraints. However, there is significant integration difficulty between increasing heterogeneous sub-models represented by various DSLs, with subsequent large designing, implementing, and maintaining costs for these DSLs as well as their supporting tools. These problems cannot be completely avoided by the second method, but they occur in a somewhat milder form. The underlying cause is that existing modeling resources, e.g., modeling infrastructure, expertise, documents, etc., are accessible to some degree.

Therefore, this paper adopts the first method (refining an existing general language) to define DSLs based on UML profiles, which captures DSM advantages and also provides many modeling resources. Model heterogeneity and loss of continuity can also be mitigated because various system models share a common language syntactic and semantic foundation.

Currently, there exist three main standard profiles released by the object management group (OMG) where some concepts can also be used in the CESS: SysML [36], schedulability, performance, time (SPT) [37], and modeling and analysis of real-time and embedded systems (MARTE) [38]. There is also the SafeML profile that is extended from a SysML subset [39]. Table 2 summarizes the profile's sources and specialties.

Table 2 Comparisons between typical UML profiles

\begin{tabular}{ccc}
\hline Name & Source & Specialty \\
\hline SysML & UML & Requirements/behavior/structure \\
SafeML & SysML & Safety-critical systems \\
SPT & UML & Schedulability/performance/time \\
MARTE & SPT & NFPs/ GRM/ Alloc/GCM/GQAM \\
\hline
\end{tabular}

Given the success of these standard UML profiles, we consider the UML profiles as the main language infrastructure to define DSLs, and propose a model driven framework to mitigate integration problems between various heterogeneous models and improve model continuity when transforming models.

\section{The model-driven framework}

This section details the proposed framework, a UML based model driven framework for developing CESS models to address model heterogeneity and continuity problems. It incorporates a domain specific profile into the four-layer metamodeling architecture within MDE. Based on the framework, a tool architecture to support the overall model development process is presented. 


\subsection{General architecture}

Fig. 2 shows the general architecture that comprises two main processes. First, meta-object facility (MOF), the four-layer metamodeling architecture, is introduced vertically to design domain specific profiles. Second, modeldriven architecture (MDA) is applied horizontally to support model transformations. Each process consists of se- veral elements with specific relationships between them. Dotted arrows represent the "instance of" relationship, where every model element has strict correspondence with a model element of the layer above. Solid arrows represent model to model (M2M) or model to text (M2T) relationships, with diagrammatic source model inputs and either diagrammatic or textual target model outputs.

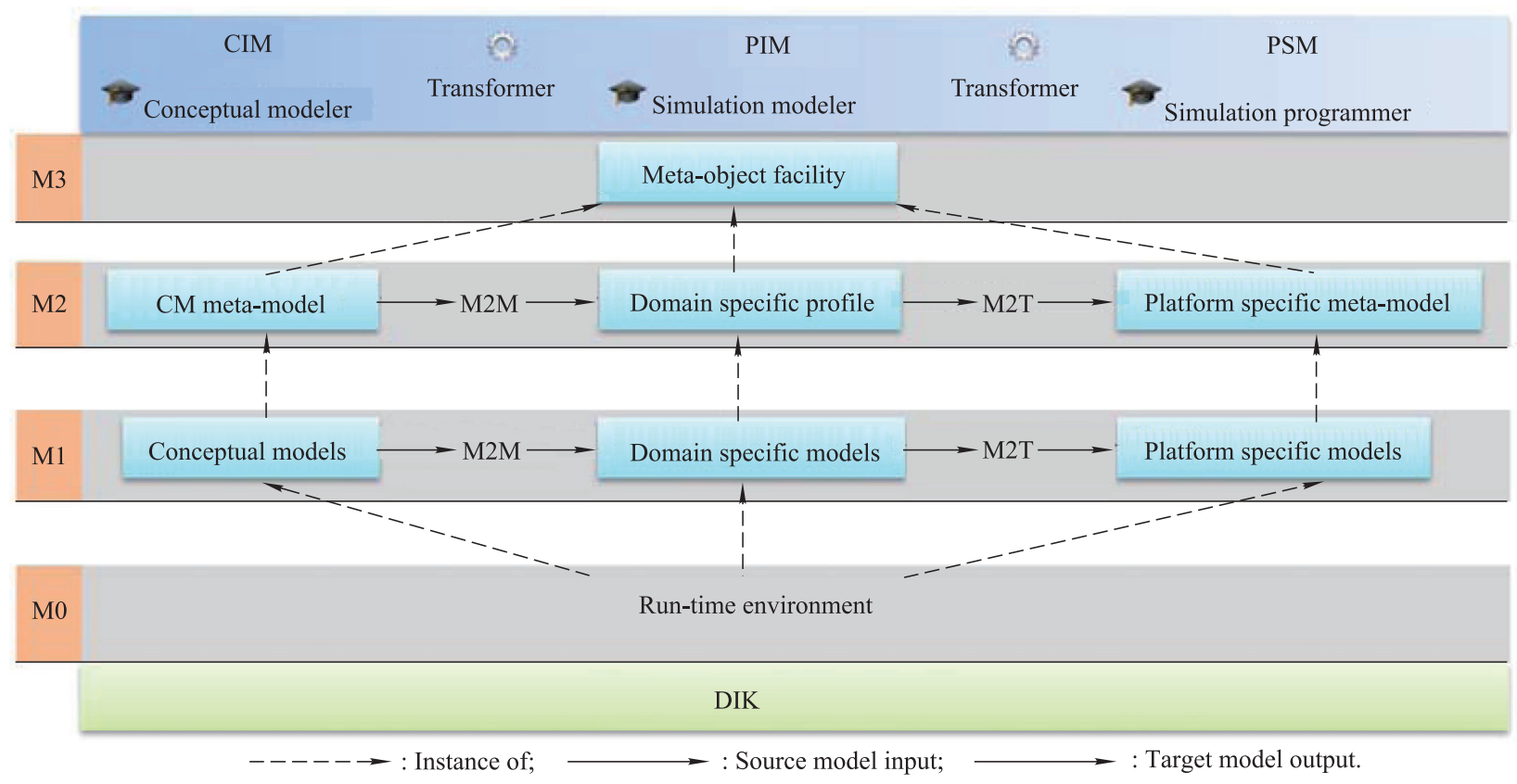

Fig. 2 General architecture of the model-driven framework within MDE

The framework attempts to address model heterogeneity and continuity problems. For model heterogeneity, a domain specific profile is developed, and model continuity is addressed by developing a set of formal model transformation rules. These two processes are closely related but mutually independent. They are related because the model transformation rules are generally expressed in the form of a metamodel, and the metamodel influences the quality of the model transformation language. They are independent since the metamodeling process is discussed from the perspective of model abstraction, whereas model transformation is discussed in the context of the model development lifecycle.

Technically, the framework is based on the UML profile. Hence, all of the models and metamodels produced share a common syntactic and semantic infrastructure. Otherwise, various products would comply with different language principles, and the model transformation rules would become complex, compounding model heterogeneity and continuity problems. In addition, UML profiles, as a functional extension of UML, have many existing tools to specify, design, implement, simulate, and analyze systems.

Furthermore, the framework introduces three model development roles, and two transform mechanism types. Conceptual independent model (CIM), platform independent model (PIM), and platform specific model (PSM) are correspondent with conceptual and simulation modelers, and simulation programmers, where later stages only can commence when developers for the former stages reach a consensus on a product. For example, once the problem owner and the conceptual modeler agree on a conceptual model, the simulation modeler can transform it into a formal model. On the other hand, M2M and M2T model transformation mechanisms are introduced as a bridge to reduce the gap between these roles.

\subsection{Incorporating domain specific profile}

\subsubsection{Applying meta-modeling architecture}

MOF is a strict four-layer metamodeling architecture in which every model element on every layer is strictly in correspondence with a model element of the layer above. Metamodeling in this framework includes a set of activi- 
ties of constructing a UML-based domain specific profile. More specifically, it mainly integrates three activities. First, the metamodeling activity specifies domain knowledge then outputs the domain specific meta-model which consists of the fundamental language constructs, relationships, and constraints. Second, the profiling activity identifies the most suitable UML meta-class which is semantically closest to the semantics of domain concepts. Third, the user friendly-modeling activity applies the domain specific profile to represent concrete applications.

\section{(i) Metamodeling}

The task of metamodeling is to capture the abstract syntax of a class of models, i.e., a modeling language. Its purpose is the initial definition of a domain model, which is the specification of what needs to be represented and how. In general, a domain model is a metamodel of a DSL that should include the following key elements.

i) A set of fundamental language constructs that represent the essential domain concepts.

ii) A set of valid relationships that exist between the domain concepts.

iii) A set of constraints that govern how the language constructs can be combined to produce valid models.

iv) The concrete syntax or notation of the language.

v) The semantics or meaning of the language.

In practice, it is not a simple task to define the above key elements, because they become woven together, and the domain model is related with the subsequent activity profiling. To obtain a valid profile with good quality, it is important to follow practical experience to help avoid common pitfalls.

Firstly, specify the domain model without any consideration of UML metamodels. This aims to acquire an unpolluted specification of the domain model. To achieve this, it is necessary to isolate domain metamodeling from the UML profiling, so domain experts and modelers can concentrate on their domains of interest based on their specialties. Unfortunately, many profile developments tend to map a domain concept or relationship into the UML metaclasses once they are identified. At first glance, this seems to be reasonable because the lightly weighted profile based method must conform to standard UML metamodels. However, the resulting domain model poorly represents the domain knowledge even though the standard UML metamodel is well satisfied.

Secondly, adjust the domain model with some loss of expressiveness if conflict occurs. Once the domain model is completed, the subsequent work cannot commence immediately without any change of the domain model. In general, the domain model may have domain specific con- straints, attributes, and relationships that conflict with standard UML metamodels. Consequently, we cannot always map the domain concepts precisely to the UML metaclasses, and we must adjust the domain model even with some loss of expressiveness if conflict occurs.

Thirdly, check the domain model to reduce the complexity of constraints. The complexity of the domain model lies in two related perspectives. The model needs to address the domain experts' requirements while complying with the UML meta model. As a result, a compromise must be worked out in a case of a conflict that may arise between the domain experts' needs and the technical modelers. For this reason, the design of the meta model can significantly impact the subsequent activities such as instantiation and constraint formulation. In some cases, domain specific constraints can become unreadable or even cannot be represented in object constraint language (OCL) [40].

(ii) UML profiling

Once metamodeling is completed, profiling starts, mapping the domain concepts to the most suitable UML base classes. There are also practical guidelines to produce suitable UML base classes for each domain concept.

i) Select UML base classes semantically similar to the domain concepts. This is important for new stereotypes to reuse existing UML tools if their semantics are most closely related. Thus, fewer domain specific constraints are required, or at least they can be more simply described.

ii) Not all the stereotypes need to come from UML base classes. Some can be constructed from super stereotypes. For example, we can obtain a stereotype directly from an existing stereotype, not necessarily extended from a specific UML metaclass.

iii) Selected UML base classes need not always align well with the domain concepts; indeed they can be contradictory. When this occurs, particular care must be taken to add appropriate constraints to resolve conflicts. For example, we can add a constraint forcing cardinality to zero for eliminating a relationship connecting some entities in the UML metaclass. If the constraint is described poorly or may be unreadable across many entities, we should consider extending another metaclass.

iv) UML profiling maps domain model elements to UML metaclasses, which requires them to be semantically aligned. The more semantic similarity, the more features could be reused, reducing language complexity. Therefore, mapping is not a one-time process, rather it is a selection that requires further investigation if the current mapping might not be the most appropriate. 
(iii) User friendly-modeling

The final important activity of language engineering is user friendly-modeling. With a customized profile, one can model using familiar language constructs. This activity not only benefits from using domain specific modeling, but also is a means to check whether the profile is qualified to represent real-world cases. In practice, we often find practical modeling issues during the use of DSLs. It is inevitable because domain specific rules are always discovered in practice even though domain experts have very professional domain knowledge.

\subsubsection{Using model transformation technology}

Model transformation is an automated process of modifying and creating one or several target models from one or several source models. The aim of model transformation is to save effort and reduce information loss as much as possible by automating model building and modification where possible. The key to designing a successful model transformation is a set of formal transformation rules to improve model continuity. Although there is no general guidance to define a good model transformation, we can evaluate model continuity according to the following criteria derived from software engineering $[41,42]$.

(i) Correctness. A model transformation is syntactically correct if the target model conforms to the target metamodel specification, and semantically correct if the target model contains as much as possible from the source model.

(ii) Completeness. A model transformation is complete if the target model has a corresponding element for each element in the source model.

(iii) Uniqueness. A model transformation is unique if there are no two identical elements in the generated target model output.

(iv) Determinism. A model transformation is determinate if it produces a uniquely defined target model output for each particular source model input.

Currently, there are two kinds of model transformation, with different expressions and output types. As the source model input or target output model of model transformations, the proposed framework defines three model types: CIM, PIM, and PSM.

On the one hand, M2M focuses on the design of a set of formal rules to ensure model continuity when transforming CIM to PIM. The transformation usually incorporates three steps.

(i) All CIM concepts, relationships, and domain specific rules are transformed into specific PIM elements, connections, and domain specific constraints, respectively.

(ii) Compare all PIM elements, connections, and con- straints to delete identical expressions.

(iii) Check the target PIM conforming to the target metamodel.

Completeness can be ensured in step i) since all CIM elements are transformed, and a corresponding PIM element can be found for each CIM element. Step ii) is helpful and necessary to reduce target PIM element redundancy. Syntactical correctness can be satisfied in step iii) since the PIM will be expressed in a given target formalism, and its semantical correctness will be evaluated in later stages of model transformation. Determinism is guaranteed implicitly in the model transformation editors, such as atlas transformation language (ATL) integrated development environment (IDE) [43]. Note that we assume the completeness of transformation from domain concepts into CIM elements is guaranteed in the proposed framework.

On the other hand, M2T transformation converts a source model into a text file. If the text is in source code form, then the transformation is also called code generation, and the transformer is also called a code generator, since the goal of the proposed framework is to generate code to support simulation, rather than supportive document creation.

The process of an M2T transformation is similar to that of an M2M transformation. The only difference is that step ii) in the M2M transformation can be skipped in an M2T transformation, since uniqueness has already been checked. Therefore, the three criteria listed above are achieved. Similarly, determinism is satisfied because model transformation editors, such as Acceleo [44], implicitly guarantee a unique output for each particular input.

\subsection{Tool support architecture}

Tool support is very important to increase software development productivity. Fig. 3 shows the tool support architecture, incorporating four layers: metamodeling environments, model editors, model transformation tools, and simulation tools [45].

In the metamodeling environment, Papyrus, a model based IDE, realizes the UML profile mechanism. The model editor layer includes the general and specific modeling environments. The former is mainly used to capture domain knowledge based on particular paradigms, such as FSMs, and the latter mainly represents domain specific models based on UML profile mechanisms. The model transformation tools include ATL for M2M transformations and Acceleo for M2T transformations. The simulation tools layer includes assistant tools integrated in the WESS platform: the data manager is a data management tool for preliminary data preparation; the scenario editor is used to describe the battle field and armed status of both 
combat sides; the cognitive behavior editor is used to support simulation analysts to specify cognitive decision behaviors; the design of experiment (DOE) editor is to design a simulation experiment, including setting the overall simulation time, selecting the experiment factors and the experiment impacts, deciding the experimental methods, etc.; visual display includes $2 \mathrm{D}$ and $3 \mathrm{D}$ viewers to free simulation developers from coding; and the output analyzer automatically generates reports based on simulation results.

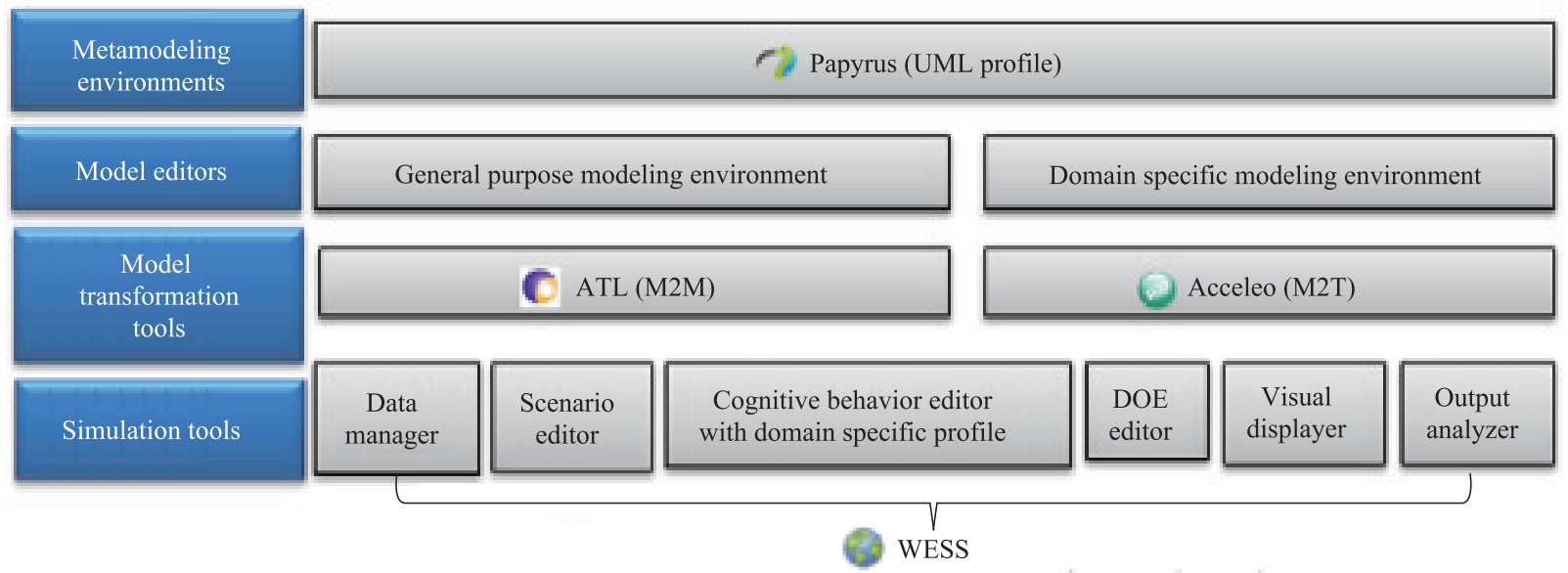

Fig. 3 Tool support architecture

\section{Torpedo behaviors design}

Torpedoes are the preferred and most effective antisubmarine weapon, with two general categories or types based on their guidance methods: wire guided and homing. Wire guided torpedoes use signals sent via thin wires, where the wire is dispensed from both the torpedo and the guidance mechanism to avoid wire stress due to their relative motion. This enables the torpedo navigational system to receive course correction commands with less interruption, such as ambient noise, which enables the launch platform to update the torpedo course to the interception point. Thus, the torpedo can reach the calculated area (target vicinity) without radiating acoustic signals. Once within the target area, the torpedo operates its internal sonar seeker and switches into the terminal attack phase.
If the wire is broken within the proximity of the target, either accidentally or intentionally, the torpedo immediately starts acoustic homing.

\subsection{Conceptual modeling}

We concentrate on torpedo's physical behavior, i.e., the behavior of a specific entity in the physical domain, which is generally independent from human will or the environment situation, and aims to capture the dynamic behavior after an entity is activated. Therefore, state and transition are two core elements of physical metamodeling.

\subsubsection{Initial representation using UML state diagram}

Fig. 4 shows how UML state diagrams can be used to describe several main physical torpedo behavior states and transitions.

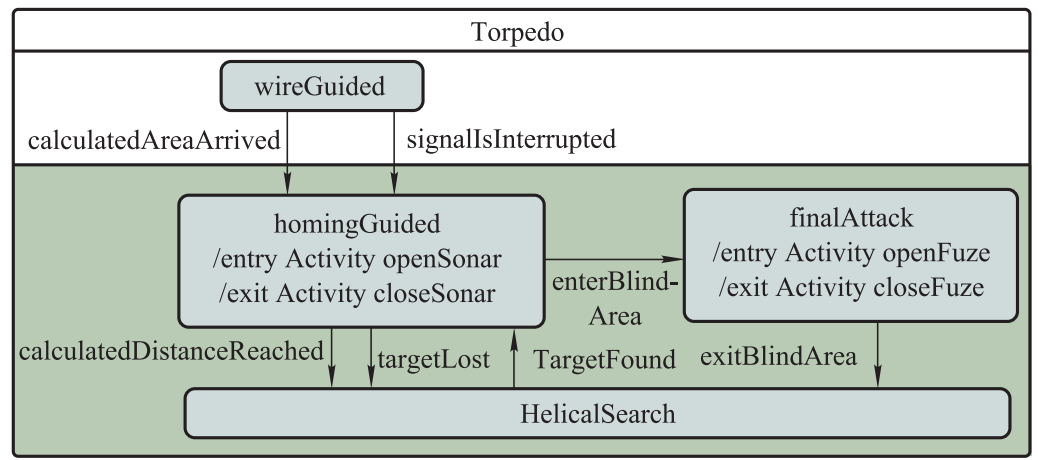

Fig. 4 Initial representation using UML state diagramF 
WireGuided torpedoes have both wire and homing guidance modes, and the transition is triggered by events from calculatedAreaArrived and signallsInterrupted. The former refers to arrival at the pre-defined area (usually the vicinity of a possible threat), and the latter is triggered when signals from the launch platform are interrupted, e.g., a broken wire. When entering the homing guidance mode, homing Guided, the torpedo's sonar (active, passive, or semi-active) opens to start homing on the target. If the target enters the torpedo's blind area, enterBlindArea, the finalAttack phase is activated, and its fuse opens. If the target subsequently exits the blind area, the fuse closes and the state switches to HelicalSearch. Within the HelicalSearch state, the torpedo switches to homingGuided if the target is detected again, i.e., targetFound, and switches back if calculatedDistanceReached or targetLost is triggered.

However, the UML state diagram is insufficient to represent some domain specific torpedo characteristics, e.g., data flow and guidance mode, etc. Therefore, we reconstruct the conceptual model considering several domain specific characteristics.

\subsubsection{Meta-modeling considering torpedo's domain specific characteristics}

Fig. 5 shows the metamodel that captures a set of torpedo's domain specific characteristics. The root element, PhysicalDiagram, incorporates four types of general information: author, createDate, description, and version. PhysicalDiagram has only one element, Element, which derives two further elements: State and Transition. State has two operations entryActivity and exitActivity, and is inherited by Pseudostate and GuidanceState. Transition may aggregate zero or more events, actions, and conditions, indicated by the diamond connection with [*] cardinality. To introduce hierarchy, State and Transition entities are derived from one Element entity, and states are allowed to contain elements. Table 3 shows details of torpedo's domain specific characteristics.

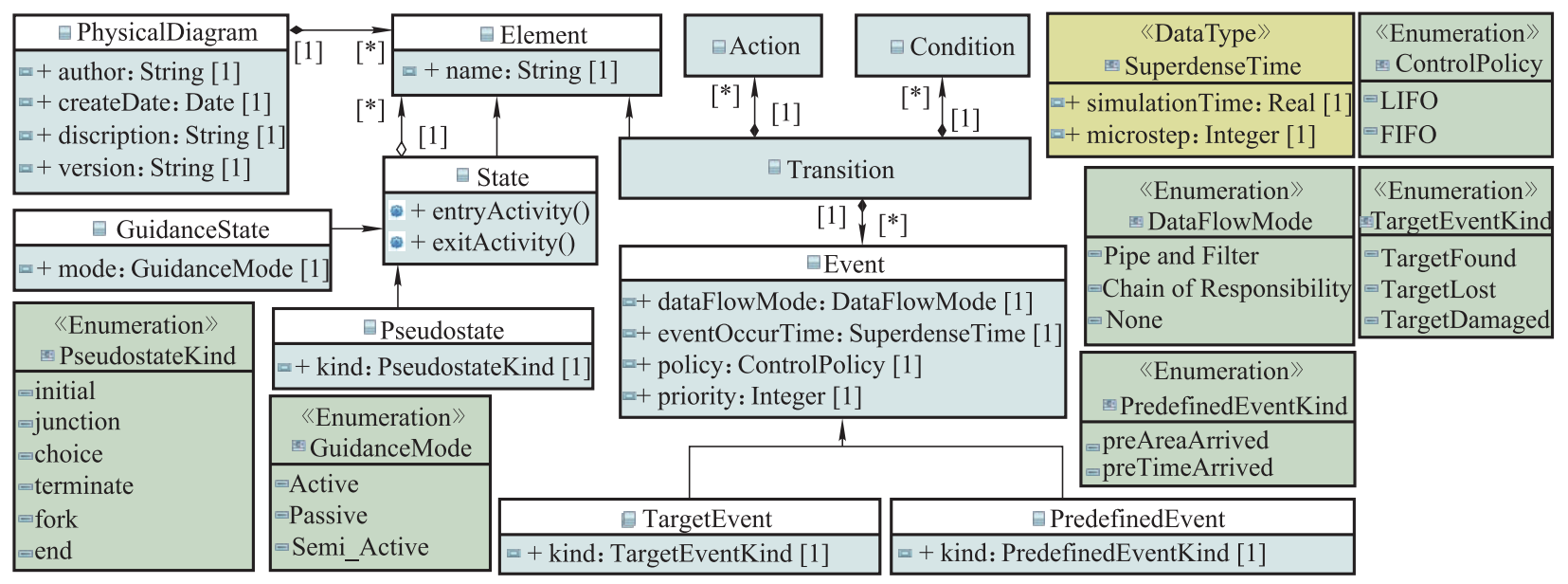

Fig. 5 Meta-model considering torpedo's domain specific characteristics

Table 3 Details of torpedo's domain specific characteristics

\begin{tabular}{cccc}
\hline Entity & Property & Type & Value \\
\hline Event & dataFlowMode & DataFlowMode (Enumeration) & Pipe and Filter, Chain of Responsibility, None \\
Event & eventOccurTime & SuperdenseTime (DataType) & simulationTime, microstep \\
Event & policy & ControlPolicy (Enumeration) & LIFO, FIFO \\
Event & priority & Integer (UML Primitive Type) & $0,1,2, \ldots$ \\
TargetEvent & kind & TargetEventKind (Enumeration) & TargetFound, TargetLost, TargetDamaged \\
PredefinedEvent & kind & PredefinedEventKind (Enumeration) & preAreaArrived, preTimeArrived \\
GuidanceState & mode & GuidanceMode (Enumeration) & Active, Passive, SemiActive \\
Pseudostate & kind & PseudostateKind (Enumeration) & initial, junction, choice, terminate, fork, end \\
\hline
\end{tabular}

One important enumeration is DataFlowMode, consisting of Pipe and Filter, Chain of Responsibility and None literals. The first literal represents data filtering to provide the required data, and the second represents that a request is processed by layers of leaders and cannot be enacted until the highest leader approves the request. The final literal is the default mode, which means no data processing is required for the event.

To specify some event occurring at the same simulation time but in different sequences, the SuperdenseTime datatype is introduced to represent casually related weakly simultaneous events. Its value is a pair $(t, n)$, called a time 
stamp, where $t$ is the simulation time and $n$ is a microstep (also called an index). In general, two time stamps $(t 1, n 1)$ and $(t 2, n 2)$ are weakly simultaneous if $t 1=t 2$, and strongly simultaneous if $n 1=n 2$ as well.

The ControlPolicy enumeration consists of LIFO and $F I F O$ literals to control how event instances are scheduled into the event queue and help ensure deterministic outcomes. With $L I F O$, events scheduled later are processed sooner, whereas the opposite applies for FIFO.

For events scheduled at the same simulation time, priority is assigned to force event instance ordering, which is a positive integer that defaults to zero.

The Event entity includes TargetEvent and PredefinedEvent events. The former has three values to represent a target being detected, undetected, or damaged, whereas the latter consists of events to represent a torpedo arriving at the predefined area or at the predefined simulation time.

The GuidanceState enumeration consists of Active, Passive, and SemiActive literals, which reflect typical guided weapon modes. The PseudostateKind enumeration has initial, junction, choice, terminate, fork, and end literals, which refer to the UML state diagram pseudo states.

There are also a set of domain specific constraints on the metamodel from two aspects.

(i) Syntactical constraints ensure the correctness of attributes, entities, and structural well formedness rules. Consider a possible syntactic constraint on the Transition entity, "One transition is able to hold a maximum of 5 events". OCL specifies this description as

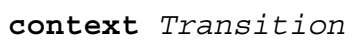

(ii) Semantic constraints specify meaningful constraints and ensure that the language satisfies them. We assume that PredefinedEvent is an event type whose data flow cannot be processed in the Pipe and Filter mode. OCL specifies this constraint as

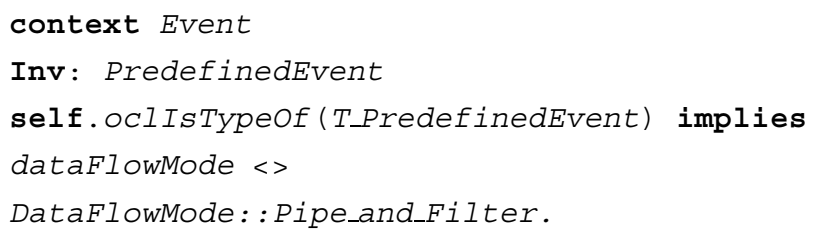

In regard of practical metamodeling experience discussed previously, many constraints on the metamodel may affect different levels of entities and concepts, which make constraint specifications complex and not straightforward to express. A key principle to reduce constraint complexity is that the models are carefully designed, or it will become difficult to define useful constraints.

\subsection{Model specification}

\subsubsection{UML profiling}

Fig. 6 shows the torpedo's domain specific profile.

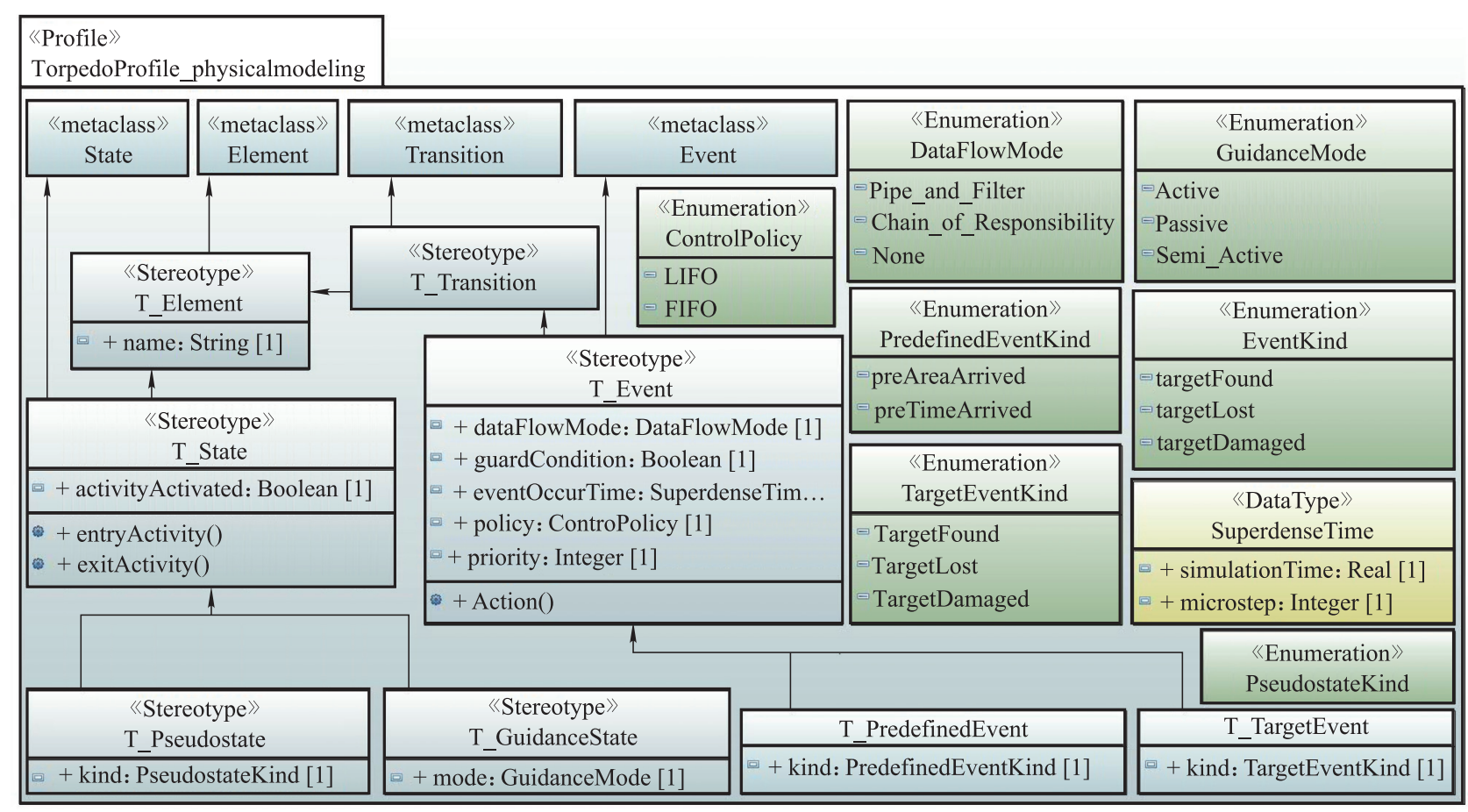

Fig. 6 Torpedo's domain specific profile 
This profile defines state and transition as core entities to describe torpedo's physical behavior. In UML, "a state models a situation during which some (usually implicit) invariant conditions hold" [46]. This is similar to the concept of the torpedo state, where a state persists for a certain time period and will not change until stimuli occur, e.g., a wire guided torpedo retains wire guidance until a wire is broken and then enters homing guidance. Therefore, a stereotype T_State is constructed to map the torpedo state to the metaclass State.

We define additional meta-information for the stereotype by adding tagged values, entryActivity() and exitActivity(), designated as its attributes or operations. Following the UML advance state concept whenever a state is entered, its entry behavior is executed before any other action is executed, and its exit behavior is executed before exiting the state.
For the other core entity, T_Transition derives T_Event, which furtherly derives T_PredefinedEvent and T_TargetEvent. In UML, “An event describes a set of possible occurrences, and an occurrence is something that has some consequence within the system" [46]. In torpedo's physical domain, an event (e.g., a target is detected) is defined as a stimulus that can cause state change. Similarly, we define a T_Event stereotype to map the torpedo event to UML meta-class Event. The stereotype has five tags: the dataFlowMode tag typed by DataFlowMode enumeration, the guardCondition tag typed by Boolean, the eventOccurTime tag typed by SuperdenseTime enumeration, the policy tag typed by ControlPolicy enumeration, and the priority tag typed by Integer. In addition, It has one Action () operation. This profile has a set of stereotypes which are enriched by a set of concrete syntaxes with some graphical notations, as listed in Table 4.

Table 4 Details of torpedo's physical profile

\begin{tabular}{|c|c|c|c|}
\hline Stereotype & Graphical notation & Meta-class & Description \\
\hline T_Element & (Abstract) & Element & $\begin{array}{l}\text { As a stereotype that derives other stereotypes of the torpedo's } \\
\text { physical profile, hierarchy is introduced. It has a tag name } \\
\text { typed by String. }\end{array}$ \\
\hline T_State & (S) & State, Element & $\begin{array}{l}\text { The core stereotype represents a physical state of torpedo that } \\
\text { will keep unchanged for a certain time period unless stimuli } \\
\text { occur. It has a Boolean tag activityActivated and two opera- } \\
\text { tions entryActivity and exitActivity. }\end{array}$ \\
\hline T_GuidanceState (Active) & & State, Element & \\
\hline T_GuidanceState (Passive) & & State, Element & $\begin{array}{l}\text { Isolating the stereotype from } T_{\text {State }} \text { stereotype aims to em- } \\
\text { phasize the guidance characteristics of torpedo. It has a tag } \\
\text { mode typed by the GuidanceMode enumeration which contains }\end{array}$ \\
\hline T_GuidanceState (Semi_Active) & (AP) & State, Element & Active, Passive, and Semi_Active modes. \\
\hline T_Pseudostate & (Similar to state diagram) & State, Element & $\begin{array}{l}\text { The stereotype refers to the pseudo states of UML state di- } \\
\text { agram, which consists of initial, junction, choice, terminate, } \\
\text { fork, and end forms. }\end{array}$ \\
\hline T_Transition & A & Transition, Element & $\begin{array}{l}\text { As another core stereotype, } T_{-} \text {Transition is used for future ex- } \\
\text { tension purpose. It derives the } T_{-} \text {Event stereotype. }\end{array}$ \\
\hline T_Event & [E] & Event, State & $\begin{array}{l}\text { The aggregation relationships fall into a stereotype. The stereo- } \\
\text { type has an Action operation and five tags: dataFlowMode, } \\
\text { guardCondition, eventOccurTime, policy and priority. }\end{array}$ \\
\hline T_TargetEvent & ET & Event, State & $\begin{array}{l}\text { The stereotype derives from T_Event, which has three kinds of } \\
\text { target events: TargetFound, TargetLost, and TargetDamaged. }\end{array}$ \\
\hline T_PredefinedEvent & EP & Event, State & $\begin{array}{l}\text { The stereotype derives from T_Event, which has two kinds of } \\
\text { predefined events: preAreaArrived and preTimeArrived. }\end{array}$ \\
\hline
\end{tabular}

In addition to the mapping rules, pre and post condition constraints are also defined. In the T_State stereotype, for example, a Boolean tag named activityActivated is added to track the situation of an activity using OCL as follows:

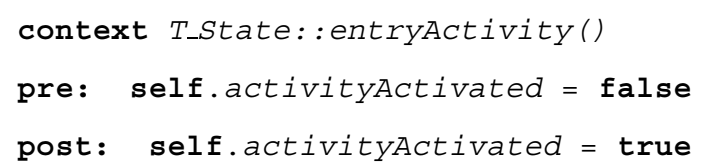

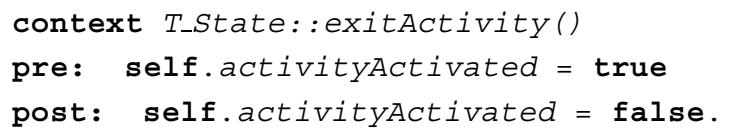

4.2.2 User friendly-modeling

Fig. 7 shows a screenshot of the domain specific modeling environment (DSME). It consists of six active windows. On the left are the project explorer, model explorer, and 


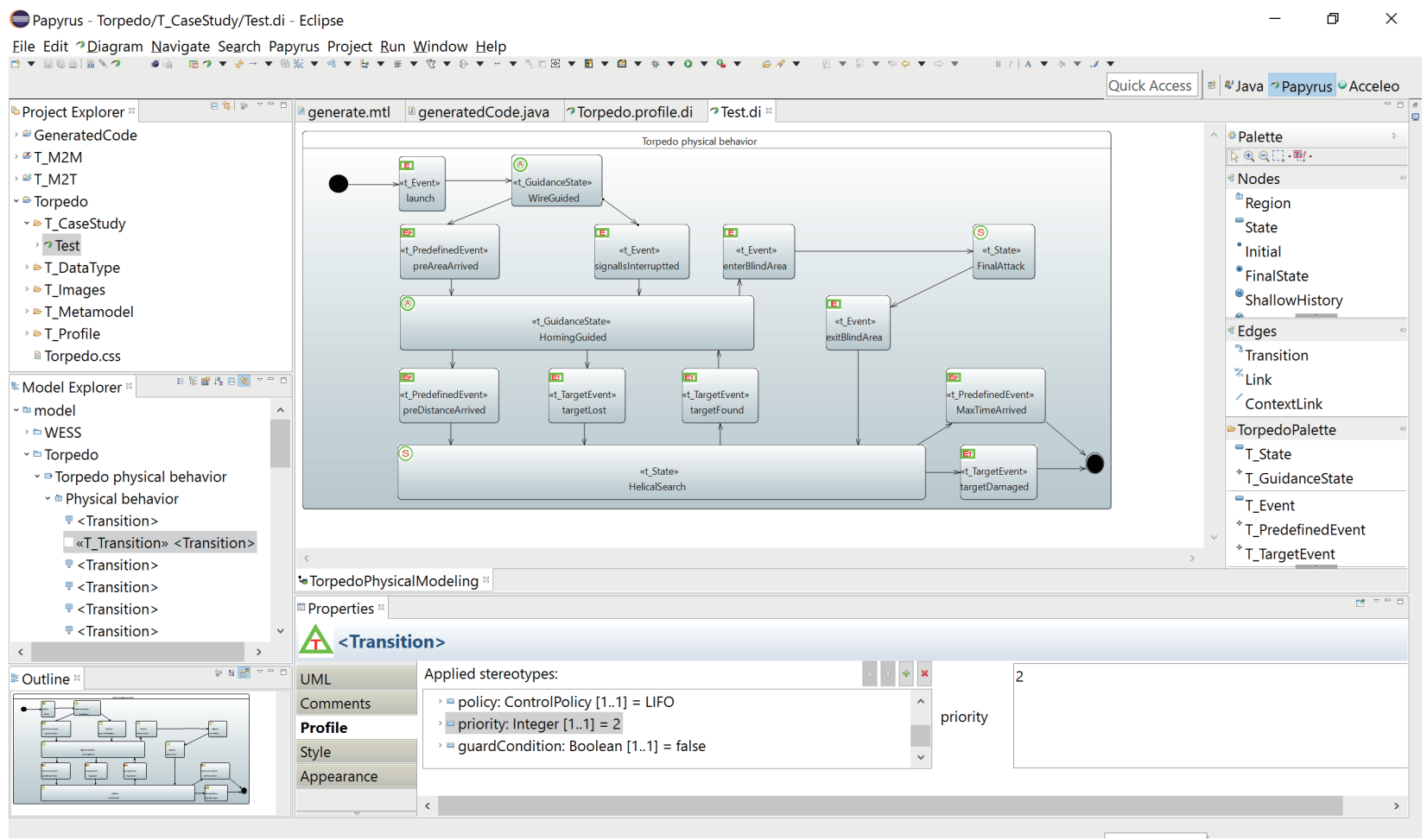

Fig. 7 Screenshot of DSME

outline; and on the right is the palette. In addition to the UML state diagram basic nodes and edges, we have added a palette named TorpedoPalette, containing T_State, T_GuidedState, T_Event, T_PredefinedEvent, T_TargetEvent, and T_Transition.

All of the domain specific considerations are incorporated in this DSME. Some have been transformed into stereotypes with prefix ' $T_{-}$', e.g., the TargetEvent entity is transformed into the T_TargetEvent stereotype; and others are specified in tag values, e.g., the priority property is specified by a tag with the same name. Several domain specific constraints are built into the DSME so that an illegal operation will incur an exception, warning, or error.

Using the DSME has several advantages over the UML state diagram.

(i) An event is regarded as an independent element. Thus, we can define more properties, e.g., priority to schedule event instance ordering.

(ii) Stereotypes are automatically applied by clicking a palette item, customized to the torpedo physical profile, as well as some predefined tagged values.

(iii) Communication between experts in different fields can be improved using customized graphical representations.

\subsection{Model implementation}

Fig. 8 shows a screenshot of the M2T transformation model (left) and its generated code framework (right). The code framework incorporates all the source model states and events that trigger state changes. It is automatically generated, but the concrete logic details accompanying each state change must be manually implemented. For example, the generated code framework implies that if the torpedo launch command is delivered when the torpedo is currently in the initial state, the torpedo will start to cruise with wire guidance. However, the code framework does not have all the necessary code definitions to support running itself, such as torpedo velocity or location changes. In practice, not every concrete detail should be considered when designing M2T transformation models, because they may heavily burden the design phase. In addition, M2T transformation model design should incorporate all the source model required information to satisfy completeness.

Following the M2T transformation process, the general source model is some instance models that must conform to a certain metamodel, and the target model can be textual, e.g., java, $\mathrm{C}++$, python, etc. Transformation model design is vital to implement the M2T transformation. This paper performs M2T transformation design using Acceleo, a template based code generator incorporating a code generation editor with syntax highlighting, completion, real time error detection, and refactoring. The source model is a collection of UML instance models, represented by the torpedo profile, and the target model is described in Java. 


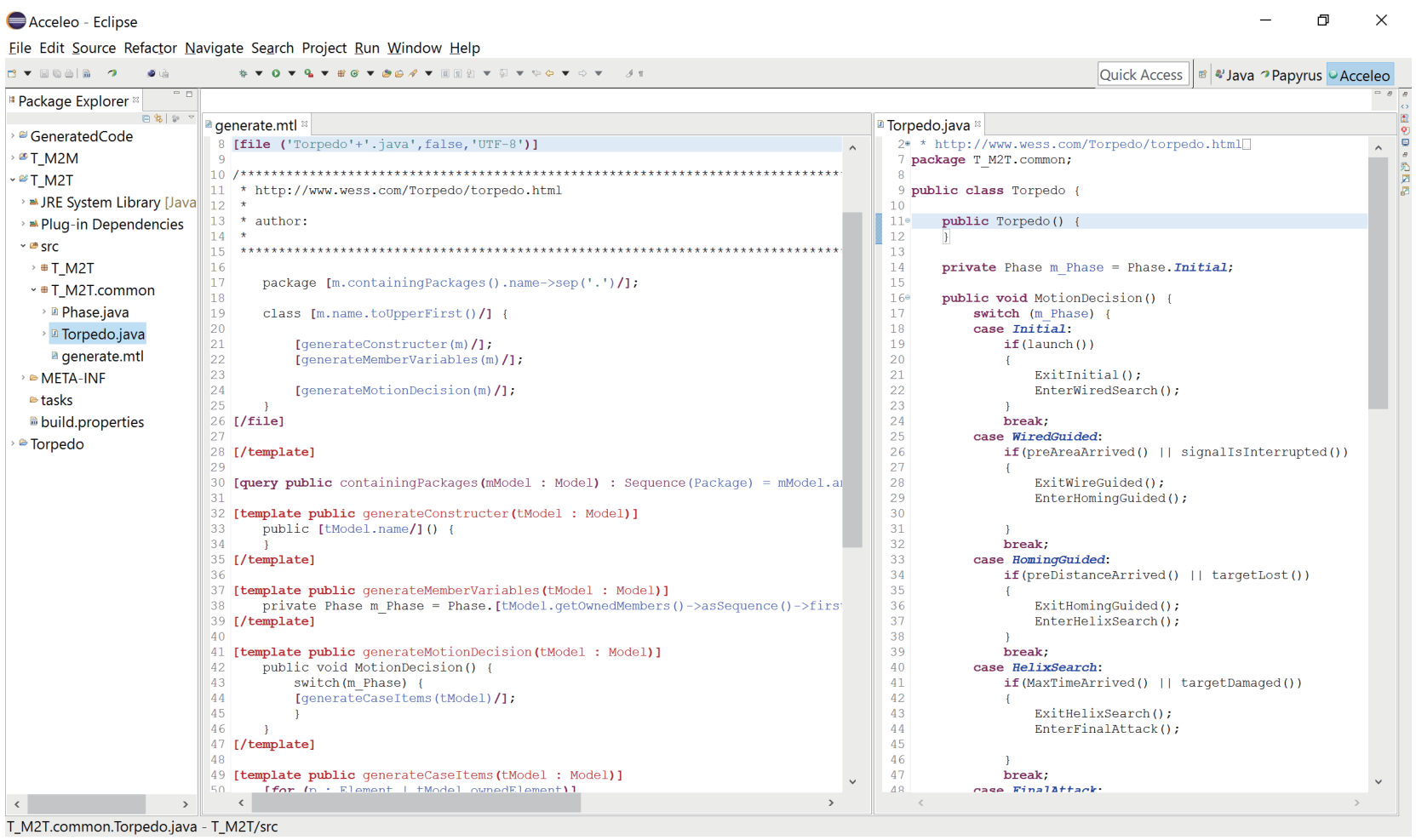

Fig. 8 Screenshot of M2T transformation model and the generated code

\section{Simulation experiment design}

Once the code is ready, a simulation expert integrates it into the WESS platform, an engagement level effectiveness simulation platform that contains a tool suite, including model editors, scenario editing, data management, script based decision modeling, experiment designer, simulator, displayer, and output analysis.

\subsection{Simulation application workflow}

Fig. 9 shows the workflow to apply the WESS system for simulation application development, incorporating three information types to prepare before running a simulation.

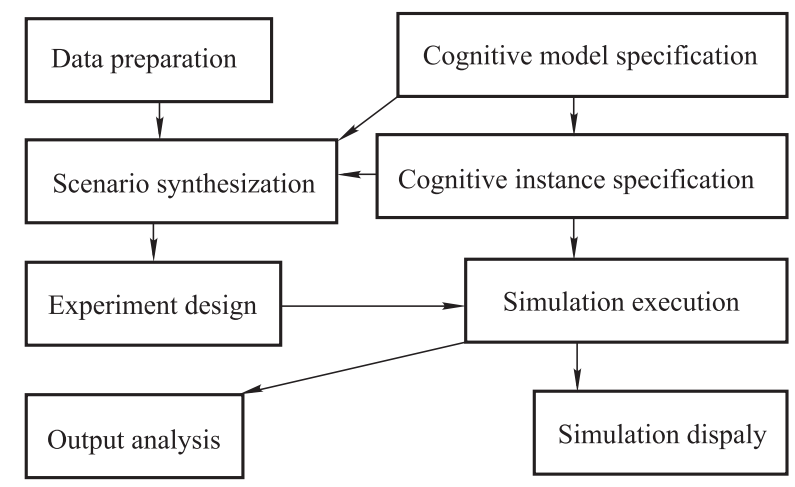

Fig. 9 Workflow of WESS application development (i) Mission scenario. Synthesizing the mission scenario automatically instantiates and composes the relevant model components within the model architecture.

(ii) Combat system data, e.g., the prototype $M K 48$ torpedo design or performance parameters. These data drive and determine how the physical behaviors are executed.

(iii) Cognitive behaviors. Separation of cognitive behavior models and corresponding simulation models allows analysts to specify cognitive behaviors in a user friendly and evolvable manner.

\subsection{Simulation results analysis}

To demonstrate the availability, consider a combat case between two sides, one that has a wire guided torpedo, and one that has no weapons, where each side has its own sonar system. In this case, we assume an interrupt signal occurs during the wire guidance phase, generated by a noisy jamming actor at random times following a Poisson process. Thus, the time between events is independent and described by identically distributed exponential variables.

We set the total logical running time for each simulation to $1000 \mathrm{~s}$ and performed 1000 rounds of Monte Carlo simulations. Fig. 10 shows the torpedo velocity and elevation data, and Table 5 shows the simulation data of torpedo's key states. 
Table 5 Simulation data of torpedo's key states

\begin{tabular}{cccccc}
\hline State & Initial & WireGuided & HomingGuided & FinalAttack & HelicalSearch \\
\hline Time/s & 468 & 471 & 511 & 795 & 809 \\
Velocity/Knot & 35.04 & 36.80 & 56.62 & 55.77 & 55.83 \\
Elevation/m & 0.00 & -15.26 & -480.00 & -479.07 & -210.24 \\
\hline
\end{tabular}

The torpedo velocity initially rises sharply then arrives at plateaus, which is similar to the elevation case, except that the torpedo increases its elevation later due to the enemy submarine's evasive strategy, e.g., up floating maneuver.

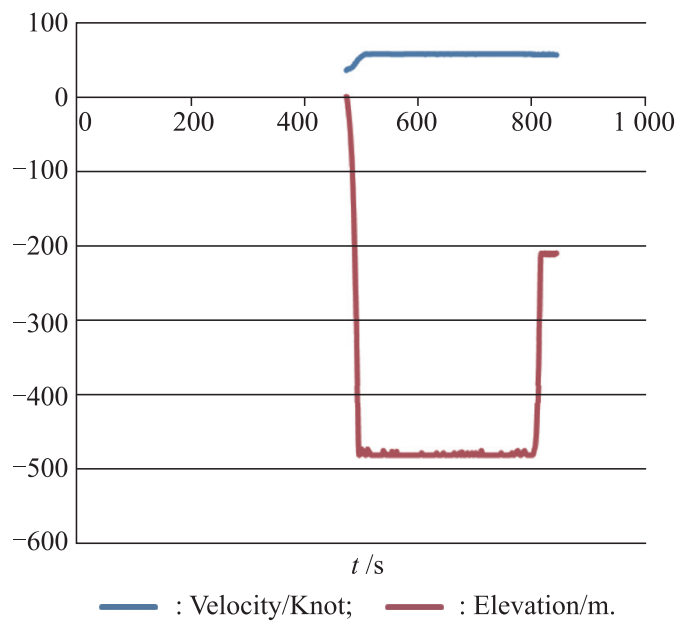

Fig. 10 Simulation curves

\section{Conclusions}

CESS incorporates multiple disciplines and rich domain knowledge, which determines its system definition often accompanied by complexity. Three decompositions of domain plus two levels of abstraction architecture has been recently used to reduce the system specification complexity. The decomposition aspect refers to static structure, physical behavior, and cognitive behavior; and the two abstraction aspects include domain invariant and application variant knowledge. A variety of $M \& S$ methods have been proposed to reduce design and development costs by reducing iteration and increasing the probability of success. Most of these methods acknowledge the importance of metamodeling and model transformation techniques, but metamodeling produces a number of modeling languages, which increase model heterogeneity. On the other hand, the poor model transformation rule reduces model continuity.

Within MDE, this paper explores a model driven framework with the objective of addressing model heterogeneity and continuity. This framework focuses on CESS modeling at the DIK level of abstraction. Consider the common language syntax and semantic foundation as well as existing tools and techniques in UML literature, a UML based domain specific profile is integrated into the framework. In this way, it provides an integrative process of the four-layer metamodeling architecture and model transformation techniques. In addition, a layered architecture of tools is also proposed to support the CESS engineering processes.

As a proof of concept, the torpedo's physical behavior is designed following the proposed framework, covering the complete development process from initial concept modeling to concrete code implementation. In this example, a UML based domain specific profile is designed to specify torpedo's physical behaviors incorporating many domain specific characteristics, that a general-purpose modeling language cannot express. A set of rules is also designed to implement M2M and M2T transformations. Consequently, the example shows mitigation of model heterogeneity by use of a common domain specific profile, and improves model continuity by use of well-defined model transformation rules. To demonstrate the availability of the generated code, a simulation experiment is performed within the WESS platform, and simulation outcomes agree well with real-world behaviors.

\section{References}

[1] LEI Y, ZHU N, YAO J, et al. Model-architecture oriented combat system effectiveness simulation. Proc. of the IEEE Winter Simulation Conference, 2015: 3190-3191.

[2] MOSTERMAN P J, VANGHELUWE H. Computer automated multi-paradigm modeling: an introduction. ACM Transactions on Modeling and Computer Simulation, 2004, 80(9): $433-$ 450.

[3] Eclipse Foundation. Eclipse Modeling Framework (EMF). http://www.eclipse.org/modeling/emf/.

[4] MARCH S T, ALLEN G N. Challenges in requirements engineering: a research agenda for conceptual modeling. Social Science and Electronic Publishing, 2009, 14(1): 157-165.

[5] Object Management Group (OMG). Business process model and notation (BPMN) version 2.0. http://www.omg.org/spec/ $\mathrm{BPMN} / \mathrm{PDF} /$.

[6] BOZLU B, DEMIRORS O. A conceptual modeling methodology: from conceptual model to design. Proc. of the Summer Computer Simulation Conference, 2008: 1-11.

[7] SMP 2.0 Metamodel (Issue 1 Revision 2: EGOS-SIM-GENTN-0100). Paris: European Space Agency (ESA), 2005.

[8] SON M J, CHO D Y, KIM T W, et al. Modeling and simulation of target motion analysis for a submarine using a script-based tactics manager. Advances in Engineering Software, 2010, 41(3): $506-516$.

[9] BOYD J R. A discourse on winning and losing (unpublished briefing slides). Maxwell AFB, AL: Air University Library, 1987.

[10] IEEE Computer Society. IEEE 1516-2010-Standard for Mo- 
deling and Simulation High Level Architecture-Framework and Rules, 2010. DOI: 10.1109/IEEESTD.2010.5953411.

[11] LEI Y L, WEI Z, XIN Z, et al. Research of SMP2-based missile countermine simulation system. Journal of System Simulation, 2009, 21(14): 4312-4316. (in Chinese)

[12] AL-AQRABAWI M S. Combat system modeling: modeling large-scale software and hardware application using UML. Blacksburg, VA: Virginia Polytechnic Institute and State University, 2001.

[13] ZHU Z, LEI Y L, ZHU N, et al. Composable modeling frameworks for networked air \& missile defense systems. NUDT Transactions, 2014, 36(5): 186 - 190. (in Chinese)

[14] BALCI O. A life cycle for modeling and simulation. Simulation, 2012, 88(7): 870-883.

[15] LI X B, YANG F, LEI Y L, et al. A model framework-based domain-specific composable modeling method for combat system effectiveness simulation. Software \& Systems Modeling, 2017, 16(4): $1201-1222$.

[16] OLIVE A. Conceptual modeling of information systems. Berlin: Springer-Verlag, 2007.

[17] HARDEBOLLE C, BOULANGER F. Exploring multiparadigm modeling techniques. Simulation, 2009, 85(11/12): $688-708$.

[18] SELIC B. A systematic approach to domain-specific language design using UML. Proc. of the IEEE International Symposium on Object and Component-Oriented Real-Time Distributed Computing, 2007: 2-9.

[19] HAREL D, RUMPE B. Meaningful modeling: what's the semantics of "semantics"? Computer, 2004, 37(10): 64-72.

[20] NORDSTROM G, SZTIPANOVITS J, KARSAI G, et al. Metamodeling - rapid design and evolution of domainspecific modeling environments. Proc. of the IEEE Conference on Engineering of Computer-Based Systems, 1999: 68-74.

[21] SEO K M, CHOI C, KIM T G, et al. DEVS-based combat modeling for engagement-level simulation. Simulation, 2014, 90(7): 759-781.

[22] LEE E A, SANGIOVANNI-VINCENTELLI A L. A framework for comparing models of computation. IEEE Trans. on Computer-Aided Design of Integrated Circuits and Systems, 1998, 17(12): $1217-1229$.

[23] PTOLEMAEUS C. System design, modeling, and simulation using Ptolemy II. Berkeley: University of California, 2014

[24] LI X B, LEI Y L, VANGHELUWE H S, et al. A multiparadigm decision modeling framework for combat system effectiveness measurement based on domain-specific modeling. Journal of Zhejiang University Science C, 2013, 14(5): 311 331.

[25] RAVINDRAN B, BARTO A G. Approximate homomorphisms: a framework for non-exact minimization in Markov decision processes. International Journal of Mineral Processing, 2004, 73(2-4): $131-144$.

[26] HU X, ZEIGLER B P. Model continuity in the design of dynamic distributed real-time systems. IEEE Trans. on Systems, Man, and Cybernetics - Part A: Systems and Humans, 2005, 35(6): $867-878$.

[27] ZEIGLER B P, PRAEHOFER H, KIM T G. Theory of modeling and simulation: integrating discrete event and continuous complex dynamic systems. 2nd ed. New York: Academic Press, 2000.

[28] Modelica Association. Modelica-A Unified Object-Oriented Language for Systems Modeling Language Specification Version 3.3. http://www.modelica.org/.

[29] SISO Base Object Model Product Development Group. Base Object Model (BOM) Template Specification. http://www.sisostds.org.

[30] European Space Agency (ESA). SMP 2.0 Handbook. http://
www.eurosim.nl/support/manuals/manual_4_2/pdf/SMP_2.0_ Metamodel-1.2.pdf.

[31] AZAR M C. Assessing the treatment of airborne tactical high energy lasers in combat simulations. Dayton: Air Force Institute of Technology, 2003.

[32] ZHU Z, LEI Y L, ZHU Y F, et al. A WESS-based method for anti-submarine simulation through planning waypoints of helicopter (WIP). Proc. of the Summer Computer Simulation Conference, 2016: 15.

[33] HALL S B, ZEIGLER B P, SARJOUGHIAN H S. Joint measure TM: distributed simulation issues in a mission effectiveness analytic simulator. Proc. of the Simulator Interoperability Workshop (SIW), 1999: 1-7.

[34] LEE E A, MESSERSCHMITT D G. Static scheduling of synchronous data flow programs for digital signal processing. IEEE Trans. on Computers, 2009, 36(1): 24-35.

[35] FENG T H, LEE E A, SCHRUBEN L W. Ptera: An eventoriented model of computation for heterogeneous systems. Proc. of 10th ACM International Conference on Embedded Software, 2010: 219-228.

[36] Object Management Group (OMG). SysML specification, version 1.2 formal. http://www.sysml.org/specs.htm.

[37] Object Management Group (OMG). UML profile for schedulable, performance, and time specification, version 1.1. http://www.omg.org/spec/SPTP/1.1/2005.

[38] Object Management Group (OMG). A UML profile for MARTE specification version 1.1. http://www.omg.org/spec/ MARTE/1.1/PDF.

[39] BIGGS G, SAKAMOTO T, KOTOKU T. A profile and tool for modelling safety information with design information in SysML. Software \& Systems Modeling, 2014, 15(1): 1-32.

[40] WARMER J, KLEPPE A. The object constraint languageprecise modeling with UML. Boston: Addison-Wesley, 1999.

[41] MENS T, GORP P V. A taxonomy of model transformation. Electronic Notes in Theoretical Computer Science, 2005, 152(1/2): $125-142$.

[42] CHALLENGER M, KARDAS G, TEKINERDOGAN B. A systematic approach to evaluating domain-specific modeling language environments for multi-agent systems. Software Quality Journal, 2016, 24(3): 755-795.

[43] JOUAULT F, ALLILAIRE F, BEZIVIN J, et al. ATL: a model transformation tool. Science of Computer Programming, 2008, 72(1/2): $31-39$.

[44] BENOUDA H, ESSBAI R, AZIZI M, et al. Modeling and code generation of android applications using acceleo. International Journal of Software Engineering and Its Applications, 2013, 10(3): $83-94$.

[45] ZHU Z, LEI Y L, SARJOUGHIAN H S, et al. Cognitive behaviors modeling using UML profile: design and experience. IEEE Access, 2017, 5: 21694-21708.

[46] Object Management Group (OMG). Unified modeling language-superstructure version 2.1.1. http://www.omg.org/ technology/documents/formal/uml.htm.

\section{Biographies}

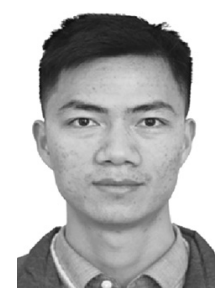

ZHU Zhi was born in 1989. He is a Ph.D. candidate in National University of Defense Technology. He was a visiting Ph.D. student at Arizona State University from 2016 to 2017 . His research interests are model-driven development and system engineering. E-mail: zhuzhi@nudt.edu.cn 


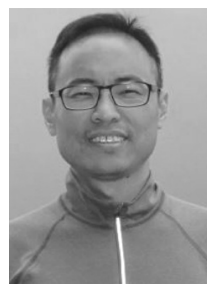

LEI Yonglin was born in 1978. He is a Ph.D. and an associate professor at National University of Defense Technology. He was a visiting scholar at Arizona State University from 2014 to 2015. His research interests are complex system modeling \& simulation, model-driven engineering, and simulation composability.

E-mail: yllei@nudt.edu.cn

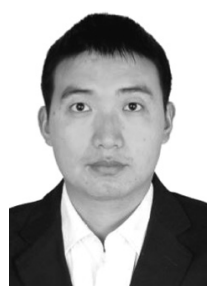

LI Xiaobo is an assistant professor at National University of Defense Technology, and also a Ph.D. candidate at University of Antwerp. His research interests are model-driven engineering techniques for M\&S, simulation based system design and demonstration, and domain-specific modeling.

E-mail: lixiaobo@nudt.edu.cn

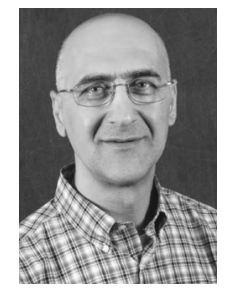

SARJOUGHIAN Hessam is an associate professor in School of Computing, Informatics, and Decision Systems Engineering at Arizona State University. He is the director of Arizona Center for Integrative Modeling and Simulation. His research interests are model composability, simulation-based design, and agent-based simulation.

E-mail: sarjoughian@asu.edu

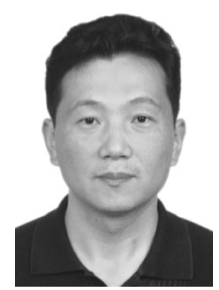

ZHU Yifan was born in 1963. He is a professor at National University of Defense Technology. He was a visiting scholar at Virginia Polytechnic Institute and State University from 2007 to 2008 . His research interests are equipment system evaluation and demonstration, and agent-based modeling and simulation.

E-mail: yfzhu@nudt.edu.cn 